\title{
Optical Identification of the Hard X-ray Source IGR J18257-0707
}

\author{
R. A. Burenin ${ }^{*}$, I. F. Bikmaev ${ }^{2,3}$, M. G. Revnivtsev ${ }^{1,4}$, J. A. Tomsick ${ }^{5}$, \\ S. Yu. Sazonov ${ }^{1,4}$, M. N. Pavlinsky ${ }^{1}$, and R. A. Sunyaev ${ }^{1,4}$ \\ ${ }^{1}$ Space Research Institute, Russian Academy of Sciences, Profsoyuznaya ul. 84/32, Moscow, 117997 Russia \\ ${ }^{2}$ Kazan State University, Kremlevskaya ul. 18, Kazan, 420008 Russia \\ ${ }^{3}$ Academy of Sciences of Tatarstan, Kazan, Tatarstan, Russia \\ ${ }^{4}$ Max-Planck-Institut für Astrophysik, Karl-Schwarzschild-Str. 1, \\ Postfach 1317, D-85741 Garching, Germany \\ ${ }^{5}$ Space Sciences Laboratory, University of California, Berkeley, California, USA \\ Received June 3, 2008
}

\begin{abstract}
We present the results of our optical identification of the hard X-ray source IGR J18257-0707 through its spectrophotometric observations with the optical RTT-150 telescope. The accurate position of the X-ray source determined using Chandra observations has allowed this source to be confidently associated with a faint optical object $\left(m_{R} \approx 20.4\right.$ ) whose optical spectrum exhibits a broad $\mathrm{H} \alpha$ emission line at redshift $z=0.037$. Thus, the source IGR J18257-0707 is a type-1 Seyfert galaxy at redshift $z=0.037$.

PACS numbers: 95.85.Kr; 95.85. Nv; 95.85.Pw; 98.54. Cm; 98.62.Tc

DOI: $10.1134 / \mathrm{S} 1063773709020017$
\end{abstract}

Key words: X-ray sources, gamma-ray sources, active galactic nuclei, optical observations.

The hard X-ray source IGR J18257-0707 was discovered during the INTEGRAL all-sky survey (Krivonos et al. 2007). This source is also known in the literature as IGR J18259-0706 (see, e.g., Stephen et al. 2006). Our group systematically carries out optical observations of unidentified INTEGRAL sources aimed at determining their nature (Bikmaev et al. 2006a, 2006b, 2008; Burenin et al. 2008; Sazonov et al. 2008; Kniazev et al. 2008). Since the optical counterpart of IGR J18257-0707 was difficult to determine, we present the results of its optical identification separately in this paper.

This source was also detected in soft X-rays in the ROSAT all-sky survey and was included in the ROSAT Bright Source Catalog under the name 1RXS J182557.5-071021 (Voges et al. 1999). The error circle for the source is about $20^{\prime \prime}$ in radius, i.e., its position is much more accurate than the INTEGRAL position of the hard X-ray source with a localization error of about $5^{\prime}$. However, this accuracy is still insufficient to unambiguously associate the source with a particular optical object (Fig. 1).

The position of the hard X-ray source was improved using the observations with the X-ray telescope onboard the Swift satellite carried out on

*E-mail: rodion@hea.iki.rssi.ru
February 12,2007 . These data give the following coordinates of the source: $\alpha, \delta=18: 25: 57.12,-07$ : $10: 24.4$ (J2000). The astrometric error is mainly systematic and is about $6 . .5$ (Moretti et al. 2006). The corresponding error circle is shown in Fig. 1.

Since the object is located at a low Galactic latitude, $b=2.35$, several stars fall within the Swift error circle due to the high star density in this region. We investigated some of these stars in the optical band. In particular, we took the spectra of a relatively bright $\left(m_{R} \approx 14.6\right)$ star falling within the error circle with the TFOSC spectrometer of the Russian-Turkish 1.5-m telescope (RTT-150) (Fig. 1). This turned out to be an ordinary K-type star. Such a star might well fall within the Swift error circle of the source by chance.

To further improve the position of the $\mathrm{X}$-ray source IGR J18257-0707, the Chandra observations of this field were carried out on February 14, 2008. the position of the source was determined with a localization error of only 0.64 (90\% confidence): $\alpha, \delta=18: 25: 57.58,-07: 10: 22.8$ (J2000). The results of the Chandra observations will be discussed in more detail in a separate paper (Tomsik et al. 2008).

The improved position of the $\mathrm{X}$-ray source IGR J18257-0707 allows it to be unambiguously associated with the optical object indicated by the 


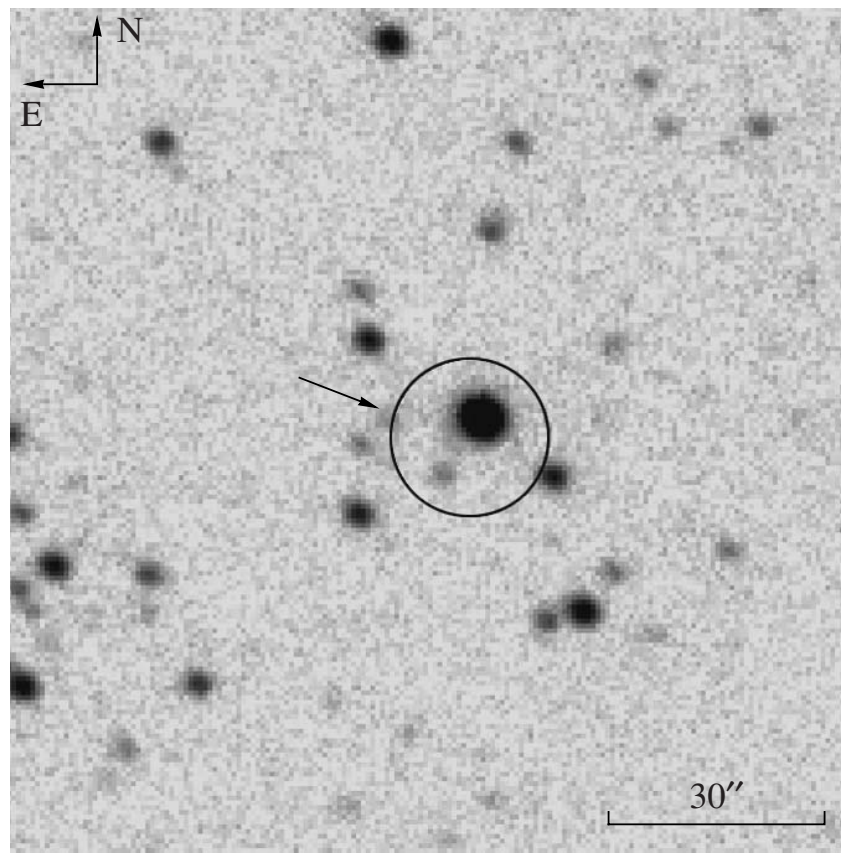

Fig. 1. $R$-band image of the field around IGR J18257-0707. The circle indicates the position of the hard X-ray source determined from Swift observations. The circle radius $\left(6^{\prime \prime} .5\right)$ shows the source localization error. The arrow indicates the object identified with the $\mathrm{X}$-ray source using Chandra observations.

arrow in Fig. 1. This object is located at an angular distance of about $7^{\prime \prime}$ from the center of the Swift error circle and its position also coincides with the position of the infrared source 2MASS J18255759-0710229 from the 2MASS survey (Cutri et al. 2003).

The magnitude of this optical object is $m_{R} \approx 20.4$ and such an object is generally believed to be too faint for spectroscopic observations with a $1.5-\mathrm{m}$ telescope. However, a $1.5-\mathrm{m}$ telescope is able to detect intense emission lines if they are present in the spectrum of the source even for such faint objects. We took the spectra of the source with a total exposure time of $6300 \mathrm{~s}$ with the RTT-150 telescope with the aim of finding such intense lines.

In these observations, we used a $100-\mu \mathrm{m}$-wide slit, which corresponds to $1^{\prime \prime} .78$ in the sky, and grism no. 15, which provides the best optical efficiency and the widest spectral coverage $(3500-9000 \AA)$. In this case, the spectral resolution was $\approx 15 \AA$ (FWHM). The data were reduced using the standard IRAF $^{1}$ software. The aperture for extracting the onedimensional spectrum of the object was specified using the spectrum of a bright nearby star that also fell on the spectrometer slit.

\footnotetext{
${ }^{1}$ http://iraf.noao.edu.
}

The spectrum obtained in these observations is shown in Fig. 2. A broad, intense emission line is clearly seen in this spectrum. The equivalent width of this line is $-276 \AA$ and its FWHM is $3600 \mathrm{~km} \mathrm{~s}^{-1}$. Only the hydrogen lines in quasar and AGN spectra can have such large widths. This cannot be a line near which other intense emission lines are observed. For example, intense narrow forbidden lines ([O III], 4959, 5007) should be observed near the $\mathrm{H} \beta$ line, while intense N V, Si IV+O IV, C IV, and other lines should be observed near the $\mathrm{L} \alpha$ line.

This should be the $\mathrm{H} \alpha$ line at redshift $z=0.037$. In that case, the object is a nearby type- 1 Seyfert galaxy; such galaxies constitute the bulk of extragalactic objects among the optical identifications of new INTEGRAL hard X-ray sources (see, e.g., Bikmaev et al. 2006a; Burenin et al. 2008). The other emission lines that are usually observed in the spectra of type-1 Seyfert galaxies are not seen in this case: the intense $\mathrm{H} \beta$ and [O III], 4959, 5007 lines lie in the blue part of the spectrum, which is much more heavily absorbed in the Galactic disk, while the narrow emission lines near $\mathrm{H} \alpha$, such as [S II], are much weaker and are unseen due to the insufficiently high signal-to-noise ratio.

The spectrum corrected for the Galactic interstellar extinction $E(B-V)=2.01$ (Schlegel et al. 1998) 

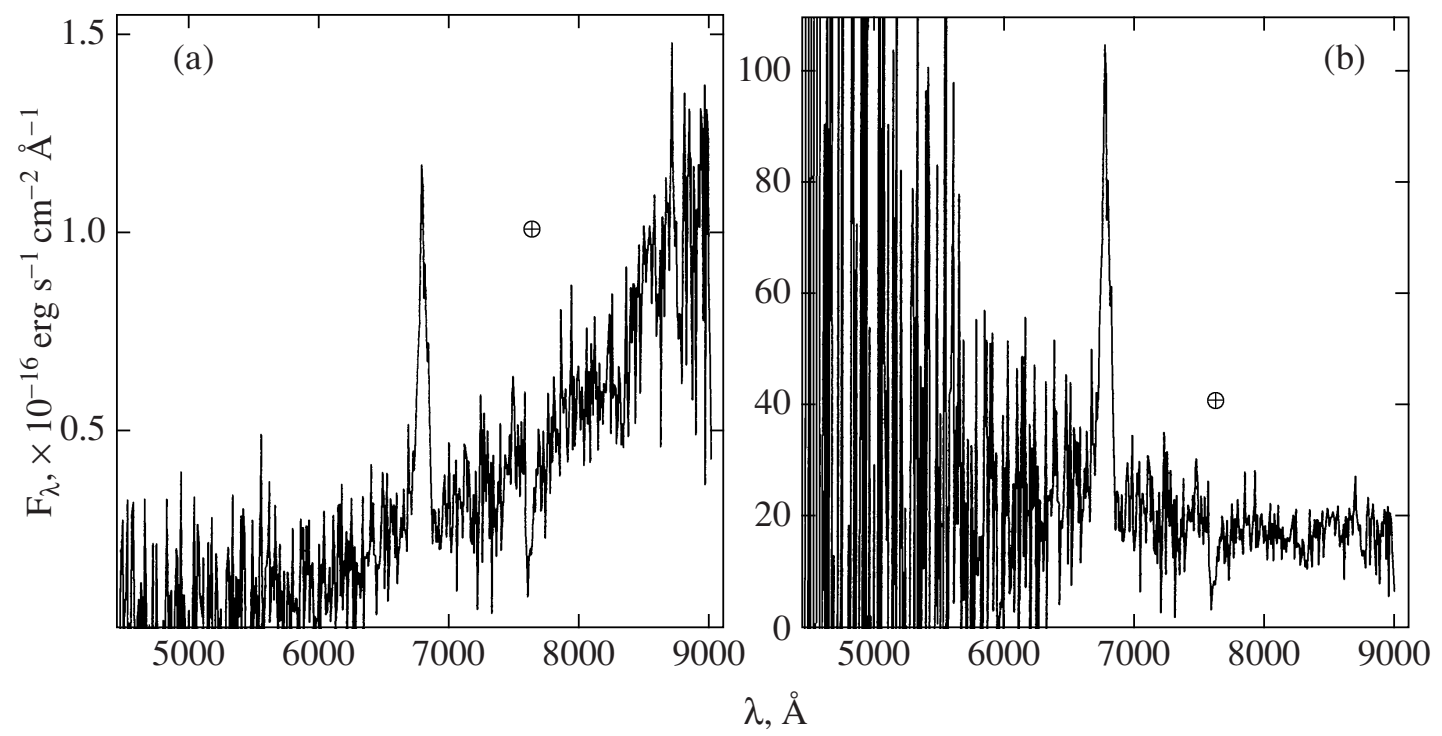

Fig. 2. (a) Spectrum of the optical object identified with the hard X-ray source IGR J18257-0707. (b) The same spectrum corrected for the Galactic extinction $E(B-V)=2.01$.

is shown in Fig. 2b. As can be seen from this figure, the overall shape of the spectrum roughly corresponds to a nonthermal continuum that is typically observed in the optical spectra of Seyfert galaxies (Elvis et al. 1994). This interstellar extinction corresponds to $A(R) \approx 5.1$, i.e., the $R$ magnitude of this object corrected for the Galactic extinction should be $\approx 15.3$. If the host galaxy of this AGN were com-

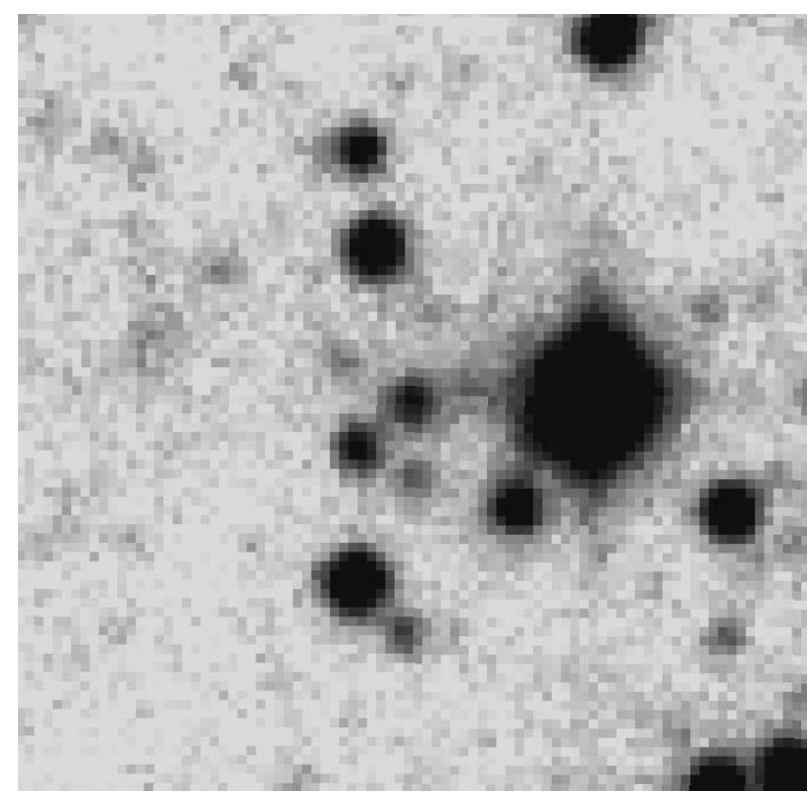

Fig. 3. $I$-band image of the AGN identified with IGR J18257-0707. parable in magnitude, then it could be seen as an extended source in the optical images. However, in our case, the extent of the object is difficult to determine reliably, because the wings of the point spread function from the nearby bright star are superimposed on the object. This is clearly seen from Fig. 3.

The column density measured along the line of sight using Swift $\mathrm{X}$-ray observations of IGR J18257-0707 is $n_{\mathrm{H}} L=(1.4 \pm 0.4) \times 10^{22} \mathrm{~cm}^{-2}$. On the other hand, with a ratio $n_{\mathrm{H}} L / E(B-V) \approx$ $4.8 \times 10^{21} \mathrm{~cm}^{2}$ typical of the Galactic interstellar matter, the reddening determined above from interstellar dust maps corresponds to a column density $n_{\mathrm{H}} L \approx 1 \times 10^{22} \mathrm{~cm}^{-2}$. The H I maps of the Galaxy (Dickey and Lockman 1990) give approximately the same column density $n_{\mathrm{H}} L \approx 0.7 \times 10^{22} \mathrm{~cm}^{-2}$. Thus, our data are consistent with the assumption that the absorption observed in $\mathrm{X}$ rays originates completely in the disk of our Galaxy.

Thus, the results of our study of IGR J18257-0707 lead us to conclude that it is an active galactic nucleus, a type- 1 Seyfert at redshift $z=0.037$.

\section{ACKNOWLEDGMENTS}

This work was supported by the Russian Foundation for Basic Research (project nos. 07-02-01004 and 08-02-00974), grants for State Support of Leading Scientific Schools (nos. NSh-4224.2008.2 and NSh-5579.2008.2), and the P-04 and OFN-17 Programs of the Russian Academy of Sciences. 


\section{REFERENCES}

1. I. F. Bikmaev, R. A. Sunyaev, M. G. Revnivtsev, and R. A. Burenin, Pis'ma Astron. Zh. 32, 250 (2006a) [Astron. Lett. 32, 221 (2006)]; astro-ph/0511405.

2. I. F. Bikmaev, M. G. Revnivtsev, R. A. Burenin, and R. A. Sunyaev, Pis'ma Astron. Zh. 32, 665 (2006b) [Astron. Lett. 32, 588 (2006)]; astro-ph/0603715.

3. I. F. Bikmaev, R. A. Burenin, M. G. Revnivtsev, S. Yu. Sazonov, R. A. Sunyaev, M. N. Pavlinsky, and N. A. Sakhibullin, Pis'ma Astron. Zh. 34, 723 (2008) [Astron. Lett. 34, 653 (2008)].

4. R. A. Burenin, A. V. Meshcheryakov, M. G. Revnivtsev, S. Yu. Sazonov, I. F. Bikmaev, M. N. Pavlinsky, and R. A. Sunyaev, Pis'ma Astron. Zh. 34, 403 (2008) [Astron. Lett. 34, 367 (2008)]; arXiv:0802.1791.

5. R. M. Cutri, M. F. Skrutskie, and S. van Dyk, IRSA 2MASS All-Sky Point Source Catalog, NASA/IPAC Infrared Science Archive (2003).

6. J. M. Dickey and F. J. Lockman, Ann. Rev. Astron. Astrophys. 28, 215 (1990).
7. M. Elvis, B. J. Wilkes, J. C. McDowell, et al., Astrophys. J. Suppl. Ser. 95, 1 (1994).

8. A. Kniazev, M. Revnivtsev, S. Sazonov et al., Astron. Telegram 1488 (2008).

9. R. Krivonos, M. Revnivtsev, A. Lutovinov, et al., Astron. Astrophys. 475, 775 (2007).

10. A. Moretti, M. Perri, M. Capalbi, et al., Astron. Astrophys. 448, L9 (2006).

11. S. Sazonov, M. Revnivtsev, R. Burenin, et al., Astron. Astrophys, in press (2008); arXive:0802.1791.

12. D. J. Schlegel, D. P. Finkbeiner, and M. Davis, Astrophys. J. 500, 525 (1998).

13. J. B. Stephen, L. Bassani, A. Malizia, et al., Astron. Astrophys. 445, 869 (2006).

14. J. A. Tomsik, S. Chaty, J. Rodriguez, et al., Astrophys. J., submitted (2008).

15. W. Voges, B. Aschenbach, Th. Boller, et al., Astron. Astrophys. 349, 389 (1999).

Translated by A. Serber 